\title{
PROBABILISTIC IDENTIFICATION OF UNSATURATED SOIL HYDRAULIC PROPERTIES FROM SINGLE RING INFILTRATION EXPERIMENT
}

\author{
E. Janouchová ${ }^{*}$ A. Kučerová ${ }^{* *}$, M. Kuráž ${ }^{* * *}$
}

\begin{abstract}
Knowledge of soil hydraulic properties is essential for modelling hydrodynamic phenomenons in soils under variably saturated conditions. This contribution focuses specifically on the properties of the top layer of soil which fundamentally influences the rainfall-runoff process, but the laboratory experimental procedures for the top-soil are very limited due to e.g. vegetation disturbing the experimental samples. The Richards equation describing the soil water flow process is here parameterised by the Mualem-van Genuchten model. The identification of the soil hydraulic parameters of this model is based on an in situ measurement of the single ring infiltration experiment. The experimental data and a priori information about parameters' values are combined according to Bayes' rule in order to obtain an updated probabilistic description of unknown parameters. The Bayesian inference is realized by the Markov chain Monte Carlo method based on a less time-consuming polynomial approximation of the Mualem-van Genuchten model.
\end{abstract}

Keywords: Soil hydraulic properties, Richards equation, Legendre polynomials, Bayesian inference, Markov chain Monte Carlo.

\section{Introduction}

The unsaturated soil hydraulic properties of the top layer of soil can be identified from the well-known single ring infiltration experiment, see Kuráž et al. (2018). The authors have presented the obstacles connected to deterministic identification based on a robust genetic algorithm caused by wide ranges of the identified parameters of the Mualem-van Genuchten model (Genuchten, 1980). The convergence issues of simulating the model itself have been solved, but nonlinearity of the numerical model leading to multimodality of the optimized objective function complicates the optimization significantly.

This contribution focuses on extension of the identification problem into the stochastic space by considering the model parameters and the observed quantity as random variables. The probabilistic approach to solving the inverse problem allows to consider uncertainties expressing the experimental errors and to evaluate the corresponding uncertainty about the estimated parameters' values (Tarantola, 2005). The core of the proposed probabilistic identification procedure is the Bayesian inference combining a priori information about the parameters' values and experimental data (Bayes and Price, 1763).

The single ring infiltration experiment and the corresponding experimental data are presented in Jačka et al. (2016). The numerical model of the experiment is described in a detail in Kuráž et al. (2018) and the core differential equation governing the water flow in a variably saturated porous medium is numerically solved in the DRUtES library (Kuráž et al., 2008, 2015). In this contribution, one of the available experimental measurement is used to demonstrate the process of probabilistic identification of the soil hydraulic parameters.

Ing. Eliška Janouchová: Faculty of Civil Engineering, CTU in Prague; Thákurova 7/2077; 166 29, Prague; CZ, eliska.janouchova@fsv.cvut.cz

** Ing. Anna Kučerová, Ph.D.: Faculty of Civil Engineering, CTU in Prague; Thákurova 7/2077; 166 29, Prague; CZ, Anna.Kucerova@cvut.cz

*** doc. Ing. Michal Kuráž, Ph.D.: Faculty of Environmental Sciences, CULS in Prague; Kamýcká 129; 165 21, Prague; CZ, kuraz@fzp.czu.cz 


\section{Parameter identification}

The purpose of this task is to identify selected parameters of soil hydraulic properties, namely van Genuchten retention curve parameters $\alpha$ and $n$, and saturated hydraulic conductivity $K_{s}$, see Tab. 1 . The model has two another parameters, which are fixed on a basis of the previous results presented in Kuráž et al. (2018). The parameter of saturated water content $\theta_{s}$ is difficult to be interpreted and in order to make the conditions of the inverse problem better, this parameter is fixed to the value 0.8 according to the expert knowledge. As it is usually done in small-scale cases (Fér and Kodešová, 2012), specific storage $S_{s}$ is set to be equal to zero here.

The expert knowledge of the parameters' values is available in a form of ranges given in Tab. 1. In the identification procedure, standardised parameters $\boldsymbol{x}$ are employed instead of the real model parameters. Each model parameter is substituted by one standardised parameter according to mutual relation defined in Tab. 1. The prior probability distribution of $\boldsymbol{x}$ is assumed to be uninformative uniform on the interval $\langle-1 ; 1\rangle$.

Tab. 1: List of model parameters with ranges of their possible values and relations to the normalized parameters to be identified.

\begin{tabular}{lccccc}
\hline \multicolumn{1}{c}{ Parameter } & Symbol & Unit & Minimum & Maximum & Relation to $x_{i}$ \\
\hline Inverse of air entry value & $\alpha$ & {$\left[\mathrm{L}^{-1}\right]$} & $10^{-4}$ & $10^{0}$ & $x_{1}=\left(\log _{10}(\alpha)+4\right) / 2-1$ \\
Pore size distribution parameter & $n$ & {$[-]$} & 1.05 & 4.00 & $x_{2}=2(n-1.05) / 2.95-1$ \\
Saturated hydraulic conductivity & $K_{s}$ & {$\left[{\left.\mathrm{~L} \cdot \mathrm{T}^{-1}\right]}^{-1}\right.$} & $10^{-3}$ & $10^{1}$ & $x_{3}=\left(\log _{10}\left(K_{s}\right)+3\right) / 2-1$ \\
\hline
\end{tabular}

The unknown parameters are inferred in the Bayesian way with a help of the Markov chain Monte Carlo (MCMC) method (Gilks et al., 2005; Spall, 2003). The numerical model of the single ring infiltration experiment is simulated with a help of the DRUtES library Kuráž et al. (2008). The model response is a curve of cumulative infiltration $I(\boldsymbol{x})$ discretised into 893 time steps $t$. In order to accelerate the sampling procedure of MCMC, each component of the full numerical model response is surrogated by its polynomial approximation.

Specifically, the metamodels are constructed from a basis of the Legendre polynomials, which are orthogonal with respect to the probability density function of uniform distribution on $\langle-1 ; 1\rangle$ (Xiu and Karniadakis, 2002). The appropriate coefficients of the polynomials are computed by regression method based on a set of model simulations for chosen input values of the model parameters (Blatman and Sudret (2010a)). At first, the design of experiments of 10.000 samples is created by latin hypercube sampling on the defined domain with respect to the prior distribution. However, many of the prior model simulations are unrealistic and out of range of the experimental data, so we utilize for the metamodel construction just a part of the obtained model simulations, as it is shown in Fig. 1. The corresponding input values of the model parameters are depicted in Fig. 2. There are obvious regions in the prior domain with no selected sample, so these regions
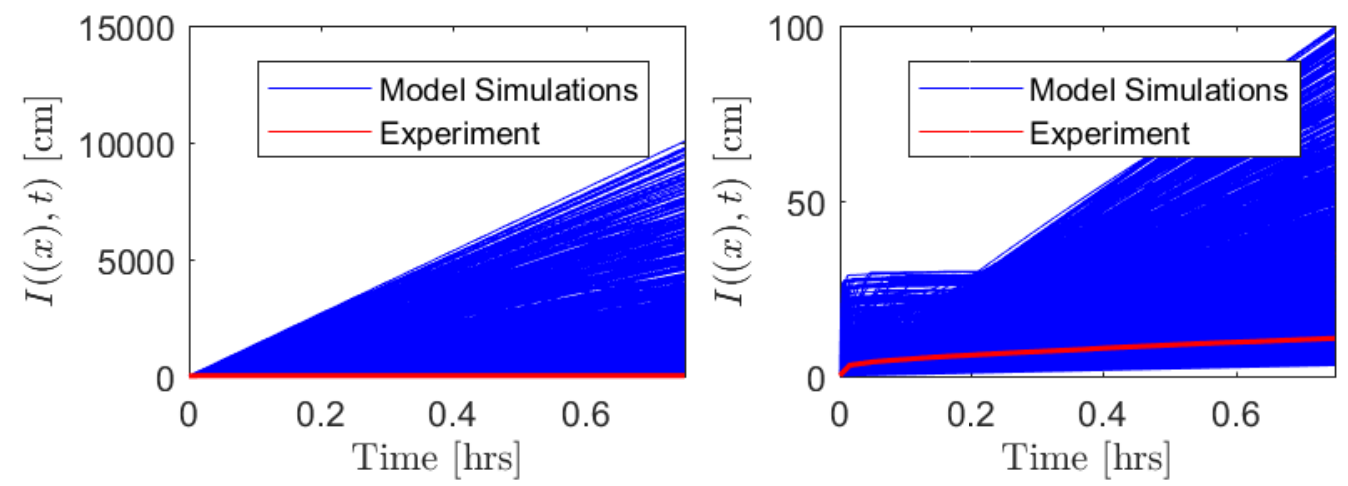

Fig. 1: Model simulations for prior parameters' distribution (left) and selection of model simulations for construction of polynomial approximation (right]. 
are not interesting in terms of the parameter identification for the specific experimental data. The restriction is particularly evident for the combinations of $x_{1}$ and $x_{3}$. The domain of the polynomial approximations is not restricted, but one has to be careful about the approximation error which is reasonable only in the region of the selected simulations. The degree of the polynomials is set to be 18 for all the time steps according to the cross-validation error based on 100 repetitions of leave-random-ten-out approach.
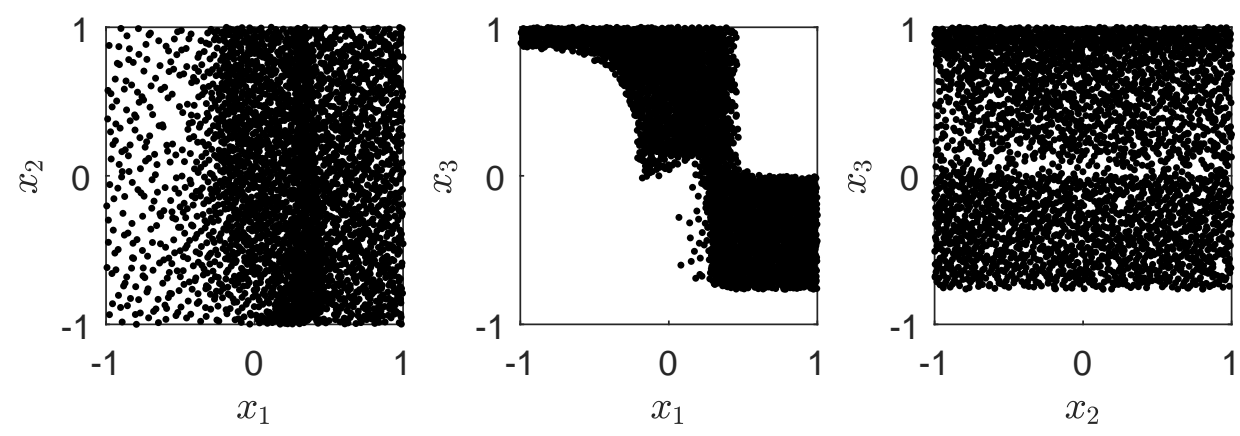

Fig. 2: Parameters' values corresponding to the selected model simulations for construction of polynomial approximation.

The likelihood function is based on the experimental errors, which are assumed as i.i.d. normal random variables with standard deviation stated by the experimenter, and theoretical errors caused by approximating the model, which are normal random variables with variances estimated from the approximation errors.

The MCMC samples of the Bayesian posterior are obtained by the Metropolis algorithm (Metropolis et al., 1953). The proposal distribution of the random step is normal with standard deviation set as to reach the acceptance rate around $40 \%$. The starting point of the random walk is equal to the input values into the model simulation with the minimal difference from the experimental curve. This setting provides a fast convergence of algorithm so as to efficiently obtain samples of the prescribed distribution. The maximum a posteriori probability (MAP) estimate of parameters' values (the mode of the posterior) and standard deviation estimated from the MCMC samples are in Tab. 2.

Tab. 2: List of model parameters with ranges of their possible values and relations to the normalized parameters to be identified.

\begin{tabular}{|c|c|c|c|c|c|}
\hline Parameter & MAP & $\operatorname{STD}\left[\cdot 10^{-4}\right]$ & Model & Parameter & MAP \\
\hline$x_{1}$ & 0.1773 & 22 & $\alpha$ & {$\left[\mathrm{L}^{-1}\right]$} & $2.26 \cdot 10^{-2}$ \\
\hline$x_{2}$ & 0.7983 & 47 & $n$ & {$[-]$} & 3.70 \\
\hline$x_{3}$ & 0.8623 & 25 & $K_{s}$ & {$\left[\mathrm{~L} \cdot \mathrm{T}^{-1}\right]$} & 5.30 \\
\hline
\end{tabular}

The results show that parameters $\alpha$ and $K_{s}$ are estimated with uncertainty twice as small as parameter $n$ according to the a posteriori variances of corresponding standardised parameters. Fig. 3 shows a good match of experimental curve and model response corresponding to MAP estimate of the model parameters, so the calibration process is successful.

\section{Conclusions}

The presented probabilistic identification procedure enables to estimate values of the soil hydraulic parameters of the Mualem-van Genuchten model together with the uncertainties associated with this estimate. The properties of the top layer of soil are identified on a basis of combining the data from the single ring infiltration experiment with expert knowledge about the parameters' values according to Bayes' rule. The a priori ranges of parameters' values are assumed wide but the analysis of the corresponding model simulations shows that there is the specific subdomain where the model captures well the physical phenomenon 

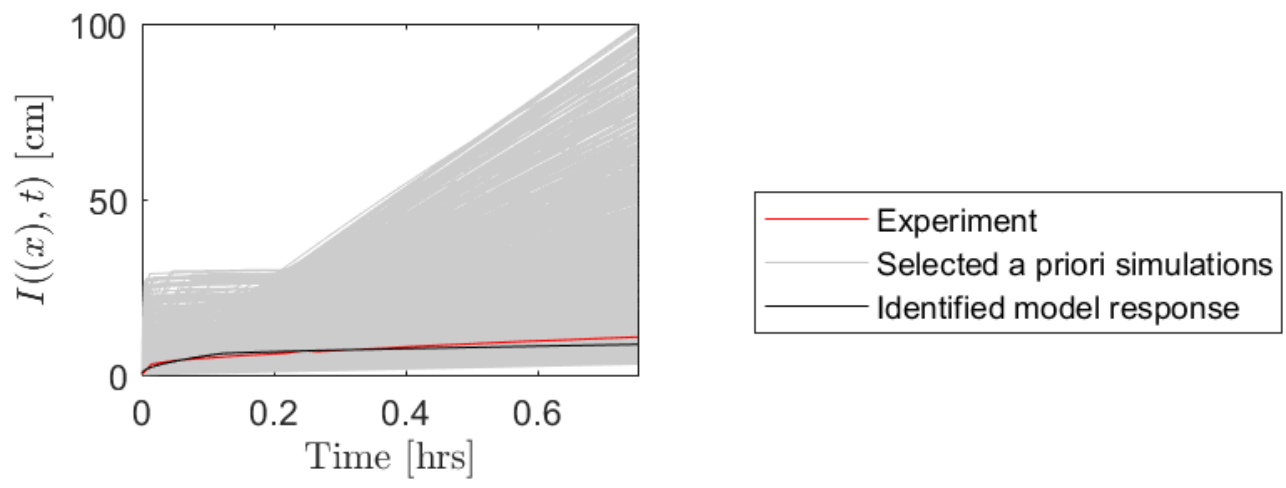

Fig. 3: Comparison of selected a priori model simulations, experimental curve and model response corresponding to identified parameters' values .

observed during the experiment. The restriction of the domain allows to construct the sufficiently accurate metamodel to be employed in the MCMC sampling in order to accelerate the identification process. The further research should be focused on updating the a priori distribution itself according to the subdomain of interest which guarantees the elimination of accidentally obtained misleading estimates caused by extrapolating of the model approximation.

\section{Acknowledgments}

The financial support of the Czech Science Foundation (Project No. 18-04262S) is gratefully acknowledged.

\section{References}

Bayes, T., Price, R. An Essay towards Solving a Problem in the Doctrine of Chances. By the Late Rev. Mr. Bayes, F. R. S. Communicated by Mr. Price, in a Letter to John Canton, A. M. F. R. S., Philosophical Transactions (1683-1775), 53:370-418, 1763.

Blatman, G., Sudret. B. An adaptive algorithm to build up sparse polynomial chaos expansions for stochastic finite element analysis. Probabilistic Engineering Mechanics, 25(2):183-197, 2010a.

Fér, M., Kodešová, R. Estimating hydraulic conductivities of the soil aggregates and their clay-organic coatings using numerical inversion of capillary rise data. Journal of Hydrology, 468-469:229-240, 2012.

Van Genuchten, M. T. A closed-form equation for predicting the hydraulic conductivity of unsaturated soils 1 Soil science society of America journal, 44(5):892-898, 1980.

Gilks, W. R., et al. Markov Chain Monte Carlo. In Encyclopedia of Biostatistics, Chichester, UK: John Wiley, 75(3):333-357, 2005.

Jačka, L., Pavlásek, J., Pech, P., Kuráž, M. Assessment of evaluation methods using infiltration data measured in heterogeneous mountain soils. Geoderma, 276:74 - 83, 2016.

Kuráž, M., Mayer, P., Blöcher, J. R. DRUtES - an opensource library for solving coupled nonlinear convectiondiffusion-reaction equations. 2008. Development version 2018.

Kuráž, M., Mayer, P., Pech, P. Solving the nonlinear and nonstationary Richards equation with two-level adaptive domain decomposition (dd-adaptivity)" Applied Mathematics and Computation, 267:207-222, 2015.

Kuráž, M., Jacčka, L., Blöcher, J. R., Lepš, M. Automated calibration methodology to avoid convergence issues during inverse identification of soil unsaturated hydraulic properties. Manuscript submitted for publication. 2018

Metropolis, N., Rosenbluth, A. W., Rosenbluth, M. N., Teller, A. H., Teller, E. Equation of state calculations by fast computing machine. The journal of chemical physics, 21:1087-1091, 1953.

Spall, J. C. Estimation via Markov Chain Monte Carlo. IEEE Control Systems Magazine, 23(2):34-45, 2003.

Tarantola, A. Inverse problem theory and methods for model parameter estimation. Philadelphia, PA: Society for Industrial and Applied Mathematics, 348 p., 2005.

Xiu, D., Karniadakis, G. E. The Wiener-Askey Polynomial Chaos for Stochastic Differential Equations. SIAM Journal on Scientific Computing, 24(2):619-644, 2002. 